\title{
perifèria
}

Número 5, Diciembre 2006

www. periferia. name

\section{Procreación, género e identidad. Debates actuales sobre el parentesco y la familia en clave transcultural}

\author{
Jorge Grau Rebollo, Departament d'Antropologia social i cultural, UAB ${ }^{1}$
}

\begin{abstract}
“En aquel Imperio, el Arte de la Cartografía logró tal Perfección que el mapa de una sola provincia ocupaba toda una Ciudad, y el mapa del Imperio toda una Provincia. Con el tiempo, esos Mapas Desmesurados no satisficieron y los Colegios de Cartógrafos levantaron un Mapa del Imperio, que tenía el tamaño del Imperio y coincidía puntualmente con él. Menos Adictas al Estudio de la Cartografía, las Generaciones siguientes entendieron que ese dilatado Mapa era Inútil y no sin Impiedad lo entregaron a las Inclemencias del Sol y de los Inviernos. En los desiertos del Oeste perduran despedazadas Ruinas del Mapa, habitadas por Animales y por Mendigos, en todo el País no hay otra reliquia de las Disciplinas Geográficas".
\end{abstract}

J orge Luis Borges

Podría muy bien decirse del parentesco que desde hace al menos treinta años está haciendo gala de una espléndida mala salud de hierro. Si en los años setenta y primeros ochenta algunos parecían anticipar su disolución, en la última década se ha constatado que no es que haya renacido de sus cenizas, como sostenía David Schneider, sino que nunca dejó de tener vigencia, pese a ciertas dudas en los procedimientos convencionales de aproximación al objeto de estudio sobre presupuestos folk mal operacionalizados $-\mathrm{y}$ prematuramente asumidos como universales- y alguna que otra mala práctica. Lo que sí ha ocurrido desde finales de los setenta (y particularmente desde mediados de los ochenta) es que la rúbrica «parentesco» ha diversificado e incorporado ópticas hasta entonces residuales $u$ oscurecidas, revelando nuevas áreas relacionales de vinculación que superaban (presuntamente) los vacíos teóricos y las objeciones empíricas de la etnografía de los últimos treinta años (relatedness, estudios de género, etc.).

\footnotetext{
${ }^{1}$ Enviar correspondencia a: jordi.grau@uab.es.
} 


\section{perifèria}

Número 5, Diciembre 2006

www. periferia.name

Por otra parte, el auge de las aproximaciones marxistas, feministas e históricas en la antropología jugaron un papel determinante en los estudios de parentesco. Las principales orientaciones teóricas en este sentido se articularon en torno a los siguientes ámbitos:

1) Destronamiento de los pilares básicos de la disciplina y hundimiento del paradigma estructural funcionalista a partir de Leach, Needham y Schneider (Peletz, 1995; Holy, 1996; Stone, 2001).

2) Incorporación de perspectivas legales, históricas y feministas (Weiner, 1976; Collier y Yanagisako, 1987; Ginsburg, 1990; Di Leonardo, 1991; Fox, 1993; Peletz, 1995).

3) Vinculación entre «parentesco», «tierra» y «propiedad» (Labby, 1977; Schneider, 1984; Holy, 1996).

4) Enfoques que explicaban la organización del parentesco como un apéndice de la economía política (Meillassoux, 1977; Godelier, 1986 [1982]).

5) Revelación del parentesco como un elemento vertebrador y reproductor de diferencias ubicado en el seno de un sistema de desigualdad estructural que permite visualizar estrategias de resistencia ante ideologías culturales dominantes (Weiner, 1992; Yanagisako y Delaney, 1995; Stone 2001).

Sabemos que desde que Morgan o Maine lo «inventaran» como parcela teórica (Needham, 1974; Kuper, 1999a y b; González Echevarría, 2000) la antropología del parentesco no ha mantenido una textura homogénea. Las líneas de interés intelectual han sido diversas y han tenido una traducción igualmente variada en el panorama etnográfico y académico. Bajo mi punto de vista, estas diversas tradiciones pueden reconstruirse a partir de una secuencia más o menos conexa de aportaciones teóricas variadas. Desde esta perspectiva, cada etapa traduce en última instancia los horizontes conceptuales, teóricos e ideológicos, que en cada contexto histórico tiene una sociedad (o al menos parte de ella) respecto a la percepción de fenómenos problemáticos o la convicción en determinados modelos de aproximación a los mismos. 


\section{perifèria}

Número 5, Diciembre 2006

www. periferia.name

No considero estas diferentes fases como ciclos teóricos excluyentes, sino como facetas o momentos teóricos parcialmente distintos (se solapan en algunas cosas, se diferencian en otras; no siempre las mismas ni por las mismas razones) fruto de acercamientos también parcialmente diferentes (en los objetivos, intereses, supuestos, hipótesis y postulados) a la realidad empírica y a la definición de dominios problemáticos desde contextos heterogéneos, historias externas variadas, que conservan en cualquier caso su valor heurístico y que son reemplazados total o parcialmente cuando las circunstancias coyunturales, a muchos niveles, varían. Así, entiendo que «linaje», «parentela», «relatedness» o «adelfia» forman parte del utillaje teórico de la antropología de la misma forma que pertenecen a él «tierra», «género» o «redistribución». Por eso, que diferentes concepciones de «linaje» puedan discrepar abiertamente es comprensible, no sólo por el marco etnográfico y el contexto en que se desarrolla-, sino también y principalmente debido al entorno histórico, ideológico e intelectual en que cada autor/a se mueve, y no necesariamente los matices de Geffray sobre «linaje», por ejemplo, pueden eliminar la repercusión de Structure and Function in Primitive Society².

Mi propósito en Procreación, género e identidad es reflexionar sobre algunos de los ámbitos más recientes que han cristalizado a partir de las improntas y las reacciones que han generado las trayectorias parcialmente convergentes de esta antropología del parentesco tan similar en algunas cosas, tan diversa en otras, y por encima de todo, tan fascinante siempre, explorando algunas de las intersecciones disciplinares más sugestivas y relevantes. Conviene hacerlo porque estos desarrollos actuales no pueden explicarse como una desherencia del pasado o como la germinación espontánea de un interés repentino por ciertos aspectos de la vida, la reproducción y la crianza. Y conviene, sobre todo, porque parafraseando a Linda Stone (1997: 6) nos estamos refiriendo a una ideología relativa a las relaciones humanas que induce nociones explicativas acerca de la creación de la

\footnotetext{
2 Véase Radcliffe-Brown, A.R. (1956 [1952]), Structure and Function in Primitive Society. Glencoe, III: Free Press.
} 


\section{perifèria}

Número 5, Diciembre 2006

\section{www.periferia.name}

humanidad y la naturaleza, así como el significado último de las conexiones biológicas y no biológicas - Stone las llama «morales»- entre personas. Se trata, pues, de dar cuenta de lo que somos, biológica y socialmente, bien en calidad de individuos aislados, bien en nuestra condición de miembros de un grupo social específico. Lo que ponemos en juego al hablar de parentesco, de familia, de género o de procreación ${ }^{3}$ es nuestra identidad como personas, como seres humanos que no actúan de modo aislado sino en compañía e imbricación con otros ${ }^{4}$. En otras palabras: cómo nos pensamos las personas y los grupos humanos entre nosotros y en relación a otros, y por qué nos (y les) pensamos precisamente así y no de otra manera.

Porque a menudo se ha interrogado al parentesco acerca de quiénes somos, quiénes podemos o vamos a ser. Sea a través de la pertenencia a grupos sociales corporativos que se estructuran sobre la base de una filiación particular y no duplicable, sea en los debates acerca del papel de la «herencia genética» en nuestra configuración como individuos, lo que tenemos siempre ante nosotros es la dimensión relacionalmente poliédrica de la identidad de las personas. Los estudios sobre parentesco y familia se han ido delineando en los últimos treinta y cinco años sobre un perfil cada vez más crítico. Se constató primero la dificultad de extrapolar conceptos para delimitar prácticas culturales diversas que, aunque pudieran parecerse en su epitelio, presentaban diferencias significativas que condicionaban la adecuación de las etiquetas importadas. Más adelante se pensó que el problema no era tanto la diversidad cultural como el utillaje analítico del que disponemos para enfrentarnos a ella. Es decir, eran nuestros propios supuestos ideológicos y

\footnotetext{
3 Entendida, como hacemos desde el Grup d'Estudi Transcultural de la Procreació (GETP) como: “la reposición de los seres humanos que (...) integran [un grupo] a través de la conceptualización y regulación de la generación, la circulación y el cuidado de los niños, la atribución de la responsabiliad última de su socialización y enculturación, hasta su madurez social y las relaciones que se desarrollan a lo largo de la vida a partir de la reproducción procreativa" (GONZÁLEZ ECHEVARRíA, A.; et al (s.d.) "Sobre la delimitación teórica de los dominios transculturales: la antropología del parentesco como teoría sociocultural de la procreación". En proceso de publicación.

${ }^{4}$ No quiero decir con ello que la identidad sea monolítica o se agote en una sola dimensión. Pero sí parece claro que una parte substancial de lo que somos refiere al ámbito de nuestra incardinación social y de las relaciones que desarrollamos a partir de ella.
} 


\section{perifèria}

Número 5, Diciembre 2006

www. periferia.name

nuestros propios modelos teóricos los que debían revisarse, no los sistemas africanos de segmentación de linajes. Supuestos y modelos que, como en el caso de «familia», se revelan extraordinariamente politéticos. A partir de mediados de los ochenta «parentesco» y «familia» se han venido encarando como conceptos polisémicos, cuya generalización transcultural debe tomarse con precaución.

A lo largo de los distintos capítulos propongo una revisión de los debates contemporáneos que afectan al parentesco - sobre todo-, y a la familia -en menor medida- en clave transcultural. Así, biología, determinismo genético, adopción u homosexualidad son ingredientes que sazonan muchas controversias en la actualidad; aunque no siempre se tiene en cuenta el contexto disciplinar en el que eclosionan ni las coyunturas varias en las que se transforman y entrelazan con el paso del tiempo.

Debe quedar claro, sin embargo, que las controversias y polémicas acerca de nuestra identidad como personas en los ámbitos que nos ocupan no se han desatado de la noche a la mañana. Conceptos como «familia», «género»o «adopción» tienen una trayectoria disciplinar concreta y se incardinan en las coyunturas políticas y sociales de maneras particulares. No aparecen por generación espontánea, ni son disciplinarmente huérfanas. No es mi objetivo aquí volver a incidir en ámbitos y periodos que ya han sido profusamente estudiados y para los cuales las publicaciones disponibles son numerosas y sumamente interesantes

Por otro lado, conviene tener presente que algunas líneas teóricas que han matizado o desafiado el hábeas clásico del parentesco no son en absoluto homogéneas en su naturaleza ni en su trayectoria (feminismos, queer theory), y que no siempre las categorías con que trabajan son tan afinadas como a veces pueda parecer (caso de «hombre» en parte de los feminismos, o de queer desde los gay and lesbian studies o la propia teoría queer). En esta línea de desafío a las nociones convencionales deben inscribirse la «adopción» ${ }^{5}$ y la circulación de niños,

\footnotetext{
${ }^{5}$ Término que mantengo entrecomillado por las razones que desarrollo en Grau Rebollo (2004 a y b) y que refieren a su presumible inconsistencia teórica cuando se aplica a la comparación cultural.
} 


\section{perifèria}

Número 5, Diciembre 2006

www. periferia. name

las nuevas tecnologías reproductivas o la surrogacy, las cuales obligan a adoptar nuevas terminologías y modificar ciertos discursos sociales. Todas ellas suponen la quiebra de la adecuación de conceptos como «paternidad», «maternidad» o «filiación» en un contexto etnográfico mucho más próximo que África o Nueva Guinea. Podría decirse que si entre los cincuenta y los setenta, «filiación» o «linaje» estuvieron en cuestión, ahora es «parentness» el que se sitúa en el ojo del huracán. Tanto en los trabajos de Pitt-Rivers, Carroll, Brady o Gudeman hace treinta años, como en la literatura más reciente sobre adopción y reproducción asistida, queda claro que la lectura social de estos fenómenos se opera desde parámetros similares, por cuanto todos estos casos constituyen conceptualmente, en nuestra sociedad, complementos a las carencias biológicas de los individuos. Una mayor atención a contextos etnográficos distintos al Europeo o Americano (Yakö, Kamano, Ndowé, etc.) muestran la especificidad de nuestras concepciones jurídicas y sociales sobre la paternidad, legitimidad e incompatibilidad de mantener al mismo tiempo dos familias diferentes. El carácter auxiliar de la adopción o las nuevas tecnologías reproductivas queda patente en la diferencia terminológica entre «tener hijos»y «adoptarlos», padres «biológicos»y «adoptivos», maternidad ( $\mathrm{y}$ paternidad) por medios naturales (diferenciados a su vez en propios y asistidos) y adoptivos, o entre madre «verdadera» y madre «desnaturalizada»; siempre sobre un contexto de pareja heterosexual, monógama, procreativa y estable. También, como recuerda Holy (1996: 166), la literatura antropológica ha reforzado el carácter foráneo de ciertos métodos de concepción o adopción refiriéndose a ellos como «parentesco ficticio», «parentesco artificial», «pseudo-parentesco» 0 parentesco «ritual».

Lo que está en juego, en definitiva, es la pertinencia de definir un marco teórico que pueda superar las limitaciones de propuestas anteriores, observando las advertencias de Needham y Schneider, sin dejarse paralizar por una lectura extrema de ninguno de los dos, especialmente del segundo. En este sentido, las diferentes teorías y orientaciones del parentesco pueden verse como un intento de 


\section{perifèria}

Número 5, Diciembre 2006

www.periferia. name

recontextualización de la disciplina, puesto que la antropología del parentesco es el resultado de combinar la observación de campo y el bagaje teórico que en cada momento ha podido adquirirse, sobre los que, en función del contexto ideológico, político y académico, se definen como dominios problemáticos. Las diversas propuestas teóricas constituyen, desde esta perspectiva, momentos distintos de investigación que se despliegan en base a tradiciones académicas y culturales concretas, delimitando objetivos de investigación particulares, que en ocasiones pretenden cubrir las carencias o áreas en blanco de aproximaciones anteriores y, en otros casos, procuran abrir nuevas vías de investigación que permitan, como sugería Lévi-Strauss, conocer un poco mejor para trabajar un poco menos mal ${ }^{6}$.

De todo lo mencionado anteriormente - unas veces a raíz de ello, y otras para diferenciarse de ello- germinan y cristalizan ámbitos de interés especial y singular en las organizaciones sociales vinculadas a nociones de biología, relacionalidad, identidad, familiaridad y parentesco que no siempre operan sobre los mismos presupuestos ni se refieren a las mismas cosas, pero que en todos los casos persiguen una mejor y más precisa comprensión del dominio problemático hacia el que se dirigen.

\section{Bibliografía}

Collier, J.; Yanagisako, S. (1987) Gender and kinship: essays toward an unified analysis. Stanford: Stanford University Press.

Di Leonardo, M. (1991) Gender at the Crossroads of Konwledge: Feminist Anthropology at the Postmodern Era. Berkeley: University of California Press.

\footnotetext{
6 “Las ciencias humanas no explican nunca, o muy raras veces, hasta el final, y no predicen sino con una seguridad limitada. Pero de este modo, comprendiendo por cuartos o por mitades, previendo una vez sobre dos o sobre cuatro, no dejan de ser menos aptas, por la íntima solidaridad que instauran entre esas semi-mitades, para otorgar a quienes la practican algo más que está a mitad de camino entre el conocimiento puro y la eficacia; la sabiduría, o por lo menos cierta forma de sabiduría que permite actuar algo menos mal porque se comprende algo mejor, pero sin poder deslindar nunca con exactitud lo que se debe a uno y otro aspecto" (Claude Lévi-Strauss, citado en González Echevarría, 1987: 215-216).
} 


\section{perifèria}

Número 5, Diciembre 2006

www.periferia. name

Fox, R. (1993) Reproduction and Succession. Studies in Anthropology, Law and Society. Londres: Transaction Publishers.

Ginsburg, F.; Tsing, A. (eds.) (1990) Uncertain Terms: Negotiating Gender in American Culture. Boston: Beacon.

Godelier, M. (1986 [1982]) La producción de los Grandes hombres. Poder y dominación masculina entre los Baruya de Nueva Guinea. Madrid: Akal Universitaria.

González Echevarría, A.; et al (s.d.) "Sobre la delimitación teórica de los dominios transculturales:la antropología del parentesco como teoría sociocultural de la procreación". En proceso de publicación.

Gonzalez Echevarría, A.

- (1987) La construcción teórica en Antropología. Barcelona: Anthropos.

- (2000) Tesis para una crítica de la singularidad cultural. Bellaterra, UAB: Servei de Publicacions d'Antropologia Cultural.

Grau Rebollo, J.

- (2004a) "Parentesco y adopción. Adoptio imitatur naturam. ¿Nature vs. nurture?"; Quaderns-e; 03, 2004a. http://www.icantropologia.org/quadernse/menu.htm.

- (2004b) "A propósito de Schneider"; Quaderns-e; 03, 2004a. http://www. icantropologia.org/quaderns-e/menu. htm.

- Holy, L. (1996) Anthropological Perspectives on Kinship. Chicago: Pluto Press.

Kuper, A. J.

- (1999a) Among the Anthropologists. History and Context in Anthropology. Londres: The Athlone Press.

- (1999b) Culture: The Anthropologist's Account. Cambridge, Mass.: Harvard University Press

Labby, D. (1977 The demistification of the Yap. Dialectics of Culture in a Micronesian Island. Chicago: Chicago University Press.

Meillassoux, Cl. (1977 [1975]) Mujeres, graneros y capitales. Madrid, S.XXI. Needham, R.

- (Ed.)(1971) Rethinking Kinship and Marriage. Londres: Tavistock Publications. 


\section{perifèria}

\section{Número 5, Diciembre 2006}

www. periferia. name

- (1974) Remarks and inventions. Skeptical Essays about Kinship. Londres: Tavistock.

Peletz, M. (1995) "Kinship Studies in Late Twentieth-Century Anthropology"; Annual Review of Anthropology; 24; pp: 343-372.

Radcliffe-Brown, A.R. (1956 [1952]), Structure and Function in Primitive Society. Glencoe, III: Free Press.

Schneider, D. M. (1984) A critique of the study of kinship. Ann Arbor: University of Michigan Press.

Stone, L.

- (1997) Kinship and Gender. Oxford: Westview Press.

- (2001) "Introduction: Theoretical Implications of New Directions in Anthropological kinship", en STONE, Linda (ed.) (2001): 1-20.

Stone, L. (Ed.) (2001) New Directions in Anthropological Kinship. Nueva York: Rowman and Littlefield Publishers Inc.

Weiner, A. (1976) Women of Value, Men of Renown: New Perspectives in trobriand Exchange. Austin: University of Texas Press.

Yanagisako, S.; Delaney, C. (eds.) (1995) Naturalizing Power. Essays in Feminist Cultural Analysis. Nueva York: Routledge. 